【研究ノート】

\title{
曲線箱桁橋の実用計算式 \\ PRACTICAL FORMULAS FOR CURVED BOX \\ GIRDER BRIDGE
}

小松 定 夫* - 林 正**

By Radao Komatsu and Masa Hayashi

\section{1. 緒言}

曲線析橋の構造解析については，すでに数編の論文を

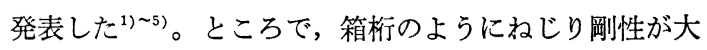
なる曲線主桁で $\alpha=R \sqrt{G J / E C_{w}}$ が比較的大きい場合に

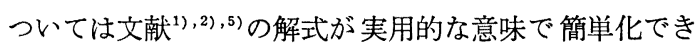
る。本文では計算の簡易化を目的として, 単純曲線箱桁 および連続曲線箱桁に関する断面力（曲げモーメント， 曲げねじりモーメント, ねじりモーメント, せん断力） および変形量（ねじり角, 断面回転角, たわみ）の実用 計算式を与えた。

本文中, 特に説明がない記号については, 文献 ${ }^{1) 22}$ 参照されたい。

\section{2. 単純支持曲線箱桁の実用計算式}

主栴断面が箱型形状のような閉断面である場合には, 文献 ${ }^{1)}$ でちえらている厳密解は 表一1,2 のようにきわ めて簡単化される。また, この近似解は実用上十分な精 度を有する。

近似解を誘導する際，つぎの条件を用いた。

1) 閉断面を有する桁では, $\alpha\left(=R \sqrt{G J / E C_{w}}\right)$ はか なり大きいので, 双曲線関数を含む項はきわめて高次の 微少項となり省略できる。

2) $\alpha^{2} \gg 1$ として，近似計算を行なった。 たとえば,

$$
\frac{\alpha^{2}}{\alpha^{2}+1} \doteqdot 1, \frac{1}{\alpha^{2}+1}-\frac{1}{\alpha^{2}} \div-\frac{1}{\alpha^{4}} \text { etc. }
$$

3）せん断中心の図心に対する偏心量は無視できるの で， $R_{0} \div R$ とした。

\section{表一1 集中荷重を受ける単純支持曲線箱桁の近似解}

\begin{tabular}{l|lll}
\hline \multirow{3}{*}{$M_{y}$} & $\bar{R} P \frac{\sin (\Phi-\psi) \sin \varphi}{\sin \Phi}$ & $(0 \leqq \varphi \leqq \Psi)$ & $(1)_{1}$ \\
& $\bar{R} P \frac{\sin (\emptyset-\varphi) \sin \Psi}{\sin \Phi}$ & $(\Psi \leqq \varphi \leqq \emptyset)$ & $(1)_{2}$ \\
\hline \multirow{3}{*}{$M_{w}$} & $\frac{R \bar{R} P \sin (\Phi-\Psi) \sin \varphi}{\alpha^{2}}$ & $(0 \leqq \varphi \leqq \Psi)$ & $(2)_{1}$ \\
& $\frac{R \bar{R} P}{\alpha^{2}} \frac{\sin (\Phi-\varphi) \sin \Psi}{\sin \emptyset}$ & $(\Psi \leqq \varphi \leqq \emptyset)$ & $(2)_{2}$ \\
\hline
\end{tabular}

* 正会員 工博 大阪大学教授 土木工学科

** 正会員 大阪大学助手 土木工学科

\begin{tabular}{|c|c|}
\hline$\theta$ & $\begin{array}{lll}\frac{R P}{G J}\left\{-\bar{R} \frac{\sin (\Phi-\Psi) \sin \varphi}{\sin \Phi}+R \frac{\Phi-\Psi}{\Phi} \varphi\right\} & (0 \leqq \varphi \leqq \Psi) & (3)_{1} \\
\frac{R P}{G J}\left\{-\bar{R} \frac{\sin (\Phi-\varphi) \sin \Psi}{\sin \Phi}+R \frac{\Phi-\varphi}{\Phi} \Psi\right\} & (\Psi \leqq \varphi \leqq \Phi) & (3)_{2}\end{array}$ \\
\hline$\beta$ & $\begin{array}{l}\frac{R \bar{R} P}{2}\left(\frac{1}{G J}+\frac{1}{E I_{y^{\prime}}}\right)\left[\frac{\sin (\Phi-\Psi)}{\sin \Phi}\left(\Phi \frac{\cos \Phi}{\sin \Phi} \sin \varphi-\varphi \cos \varphi\right)\right. \\
\left.\quad+\{\sin (\Phi-\Psi)-(\Phi-\Psi) \cos (\Phi-\Psi)\} \frac{\sin \varphi}{\sin \Phi}\right] \\
(0 \leqq \varphi \leqq \Psi) \quad(4)_{1} \\
\frac{R \bar{R} P}{2}\left(\frac{1}{G J}+\frac{1}{E I_{y^{\prime}}}\right)\left[\frac{\sin (\Phi-\varphi)}{\sin \Phi}\left(\Phi \frac{\cos \Phi}{\sin \Phi} \sin \Psi-\Psi \cos \Psi\right)\right. \\
\left.\quad+\{\sin (\Phi-\varphi)-(\Phi-\varphi) \cos (\Phi-\varphi)\} \frac{\sin \Psi}{\sin \Phi}\right] \\
(\Psi \leqq \varphi \leqq \Phi) \quad(4)_{2}\end{array}$ \\
\hline$\delta$ & $\begin{array}{l}R^{2} P\left[\frac{R}{G J}\left\{-\frac{\sin (\Phi-\Psi) \sin \varphi}{\sin \Phi}+\frac{\Phi-\Psi}{\Phi} \varphi\right\}-\frac{\bar{R}}{2}\left(\frac{1}{G J}+\frac{1}{E I_{y^{\prime}}}\right)\right. \\
\quad \times\left\{\frac{\sin (\Phi-\Psi)}{\sin \Phi}\left(\Phi \frac{\cos \Phi}{\sin \Phi} \sin \varphi-\varphi \cos \varphi\right)+(\sin (\Phi-\Psi)\right. \\
\left.\left.\quad-(\Phi-\Psi) \cos (\Phi-\Psi)) \frac{\sin \varphi}{\sin \Phi}\right\}\right] \quad(0 \leqq \varphi \leqq \Psi) \quad(5)_{1} \\
R^{2} P\left[\frac{R}{G J}\left\{-\frac{\sin (\Phi-\varphi) \sin \Psi}{\sin \Phi}+\frac{\Phi-\varphi}{\Phi} \Psi\right\}-\frac{\bar{R}}{2}\left(\frac{1}{G J}+\frac{1}{E I_{y^{\prime}}}\right)\right. \\
\quad \times\left\{\frac{\sin (\Phi-\varphi)}{\sin \Phi}\left(\Phi \frac{\cos \Phi}{\sin \Phi} \sin \Psi-\Psi \cos \Psi\right)+(\sin (\Phi-\varphi)\right. \\
\left.\left.\quad-(\Phi-\varphi) \cos (\Phi-\varphi)) \frac{\sin \Psi}{\sin \Phi}\right\}\right] \quad(\Psi \leqq \varphi \leqq \Phi) \quad(5)_{2}\end{array}$ \\
\hline$T_{s}$ & $\begin{array}{l}-P\left\{\bar{R} \frac{\sin (\Phi-\Psi) \cos \varphi}{\sin \Phi}-R \frac{\Phi-\Psi}{\Phi}\right\} \\
P\left\{\bar{R} \frac{\cos (\Phi-\varphi) \sin \Psi}{\sin \Phi}-R \frac{\Psi}{\Phi}\right\}\end{array}$ \\
\hline$T_{w}$ & $\begin{array}{l}-\frac{\bar{R} P}{\alpha^{2}} \frac{\sin (\Phi-\Psi) \cos \varphi}{\sin \Phi} \\
\frac{\bar{R} P}{\alpha^{2}} \frac{\cos (\Phi-\varphi) \sin \Psi}{\sin \Phi}\end{array}$ \\
\hline$T$ & $\begin{array}{l}-P\left\{\bar{R} \frac{\sin (\Phi-\Psi) \cos \varphi}{\sin \Phi}-R \frac{\Phi-\Psi}{\Phi}\right\} \\
P\left\{\bar{R} \frac{\cos (\Phi-\varphi) \sin \Psi}{\sin \Phi}-R \frac{\Psi}{\Phi}\right\}\end{array}$ \\
\hline$Q$ & $\begin{array}{l}(0 \leqq \varphi \leqq \Psi) \quad(9)_{1} \\
(\Psi \leqq \varphi \leqq \emptyset) \quad(9)_{2}\end{array}$ \\
\hline $\bar{Q}$ & $\begin{array}{l}\frac{\bar{R} P}{R} \frac{\sin (\Phi-\Psi) \cos \varphi}{\sin \Phi} \\
-\frac{\bar{R} P}{R} \frac{\cos (\Phi-\varphi) \sin \Psi}{\sin \Phi}\end{array}$ \\
\hline
\end{tabular}

表一2 集中トルクを受ける単純支持曲線箱桁の近似解

\begin{tabular}{l|ccc}
\hline \multirow{3}{*}{$M_{y}$} & $-m_{T} \frac{\sin (\emptyset-\Psi) \sin \varphi}{\sin \Phi}$ & $(0 \leqq \varphi \leqq \Psi)$ & $(11)_{1}$ \\
& $-m_{T} \frac{\sin (\emptyset-\varphi) \sin \Psi}{\sin \Phi}$ & $(\Psi \leqq \varphi \leqq \emptyset)$ & $(11)_{2}$ \\
\hline
\end{tabular}




\begin{tabular}{|c|c|c|}
\hline$M_{w}$ & $\begin{array}{l}-m T \frac{R}{\alpha^{2}} \frac{\sin (\Phi-\Psi) \sin \varphi}{\sin \Phi} \\
-m_{T} \frac{R}{\alpha^{2}} \frac{\sin (\Phi-\varphi) \sin \Psi}{\sin \Phi}\end{array}$ & $\begin{array}{ll}(0 \leqq \varphi \leqq \Psi) & (12)_{1} \\
(\Psi \leqq \varphi \leqq \emptyset) & (12)_{2}\end{array}$ \\
\hline$\theta$ & $\begin{array}{l}m_{T} \frac{R}{G J} \frac{\sin (\Phi-\Psi) \sin \varphi}{\sin \Phi} \\
m_{T} \frac{R}{G J} \frac{\sin (\Phi-\varphi) \sin \Psi}{\sin \Phi}\end{array}$ & $\begin{array}{ll}(0 \leqq \varphi \leqq \Psi) & (13)_{1} \\
(\Psi \leqq \varphi \leqq \emptyset) & (13)_{2}\end{array}$ \\
\hline$\beta$ & $\begin{array}{r}-m_{T} R\left[\frac{1}{2}\left(\frac{1}{G J_{t}}+\frac{1}{E I_{y}}\right)\{\right. \\
+(\sin (\Phi-\Psi)-(\Phi-\Psi) \\
-\frac{1}{G J} \frac{\sin (\Phi-\Psi) \sin \varphi}{\sin \Phi} \\
-m_{T} R\left[\frac{1}{2}\left(\frac{1}{G J}+\frac{1}{E I_{y^{\prime}}}\right)\{\right. \\
+(\sin (\Phi-\varphi)-(\Phi-\varphi) \\
-\frac{1}{G J} \frac{\sin (\Phi-\varphi) \sin \Psi}{\sin \Phi}\end{array}$ & 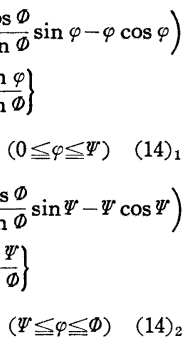 \\
\hline$\delta$ & $\begin{array}{l}\frac{m_{T} R^{2}}{2}\left(\frac{1}{G J}+\frac{1}{E I_{y^{\prime}}}\right)\left[\frac{\sin (}{\sin }\right. \\
\quad+\{\sin (\Phi-\Psi)-(\Phi-\Psi) \\
\frac{m_{T} R^{2}}{2}\left(\frac{1}{G J}+\frac{1}{E I_{y^{\prime}}}\right)\left[\frac{\sin (}{\sin }\right. \\
\quad+\{\sin (\Phi-\varphi)-(\Phi-\varphi)\end{array}$ & $\begin{array}{l}\operatorname{in} \varphi-\varphi \cos \varphi) \\
\varphi \varphi \\
\left.\frac{\ln }{\varphi}\right] \\
(0 \leqq \varphi \leqq \Psi) \quad(15)_{1} \\
\operatorname{in} \Psi-\Psi \cos \Psi) \\
\Psi \\
\Phi] \\
(\Psi \leqq \varphi \leqq \Phi) \quad(15)_{2}\end{array}$ \\
\hline$T_{s}$ & $\begin{array}{l}m_{T} \frac{\sin (\Phi-\Psi) \cos \varphi}{\sin \Phi} \\
-m_{T} \frac{\cos (\Phi-\varphi) \sin \Psi}{\sin \Phi}\end{array}$ & $\begin{array}{l}(0 \leqq \varphi \leqq \Psi) \quad(16)_{1} \\
(\Psi \leqq \varphi \leqq \Phi) \quad(16)_{2}\end{array}$ \\
\hline$T_{w}$ & $\begin{array}{l}\frac{m_{T}}{\alpha^{2}} \frac{\sin (\Phi-\Psi) \cos \varphi}{\sin \Phi} \\
-\frac{m_{T}}{\alpha^{2}} \frac{\cos (\Phi-\varphi) \sin \Psi}{\sin \Phi}\end{array}$ & $\begin{array}{ll}(0 \leqq \varphi \leqq \Psi) & (17)_{1} \\
(\Psi \leqq \varphi \leqq \emptyset) & (17)_{2}\end{array}$ \\
\hline$T$ & $\begin{array}{l}m_{T} \frac{\sin (\Phi-\Psi) \cos \varphi}{\sin \Phi} \\
-m_{T} \frac{\cos (\Phi-\varphi) \sin \Psi}{\sin \Phi}\end{array}$ & $\begin{array}{l}(0 \leqq \varphi \leqq \Psi) \quad(18)_{1} \\
(\Psi \leqq \varphi \leqq \Phi) \quad(18)_{2}\end{array}$ \\
\hline$Q$ & 0 & (19) \\
\hline $\bar{Q}$. & $\begin{array}{l}-\frac{m_{T}}{R} \frac{\sin (\Phi-\Psi) \cos \varphi}{\sin \Phi} \\
\frac{m_{T}}{R} \frac{\cos (\emptyset-\varphi) \sin \Psi}{\sin \Phi}\end{array}$ & $\begin{array}{l}(0 \leqq \varphi \leqq \Psi) \\
(\Psi \leqq \varphi \leqq \Phi)\end{array}$ \\
\hline
\end{tabular}

ほぼ 1 軸対称で, $I_{y z} \doteqdot 0$ である箱桁断面の忘力は, 次 式のようになる。ここで座標系は図一1のようにとるも のとする。

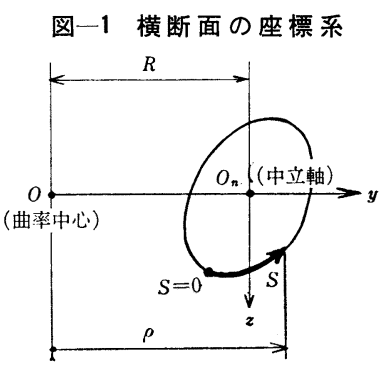

垂直応力

$$
\sigma=\frac{R}{\rho} \frac{M_{y}}{I_{y}} z+W_{s} \frac{M_{w}}{C_{w}}
$$

\section{3. 連続曲線箱桁の実用計算式}

主桁断面が箱型断面である連続曲線箱桁の実用計算式 は, 前節で近似解を導いたのと同じ条件を用いて, 文献 5）の諸式よりつぎのようになる。

\section{（1）弾性方程式の係数}

$$
\begin{aligned}
& {[a]_{m j}=\frac{R_{j}}{E_{s} I_{y^{\prime}, j}}\left(\frac{2 \Phi_{j}-\sin 2 \Phi_{j}}{4 \sin ^{2} \Phi_{j}}\right)+\frac{R_{j}}{G_{s} J_{j}}} \\
& \times\left(\frac{\Phi_{j}}{2} \operatorname{cosec}^{2} \Phi_{j}+\frac{1}{2} \cot \Phi_{j}-\frac{1}{\Phi_{j}}\right) \\
& a_{m, m-1}=\frac{R_{m-1}}{E_{s} I_{y}{ }^{\prime}, m-1}\left(\frac{\sin \Phi_{m-1}-\Phi_{m-1} \cos \Phi_{m-1}}{2 \sin ^{2} \Phi_{m-1}}\right) \\
& -\frac{R_{m-1}}{G_{s} J_{m-1}}\left(\frac{1}{2}\left[\Phi_{m-1} \cot \Phi_{m-1}+1\right]\right. \\
& \left.\times \operatorname{cosec} \Phi_{m-1}-\frac{1}{\Phi_{m-1}}\right) \\
& a_{m, m+1}=\frac{R_{m}}{E_{s} I_{y}{ }^{\prime}, m}\left(\frac{\sin \Phi_{m}-\Phi_{m} \cos \Phi_{m}}{2 \sin ^{2} \Phi_{m}}\right)-\frac{R_{m}}{G_{s} J_{m}} \\
& \times\left(\frac{1}{2}\left[\Phi_{m} \cot \Phi_{m}+1\right] \operatorname{cosec} \Phi_{m}-\frac{1}{\Phi_{m}}\right) \\
& {[b]_{m j}=-\frac{1}{G_{s} J_{j}}\left(\cot \Phi_{j}+\frac{1}{\alpha_{j}}-\frac{1}{\Phi_{j}}\right)} \\
& b_{m, m-1}=\frac{1}{G_{s} J_{m-1}}\left(\operatorname{cosec} \Phi_{m-1}-\frac{1}{\Phi_{m-1}}\right) \\
& b_{m, m+1}=\frac{1}{G_{s} J_{m}}\left(\operatorname{cosec} \Phi_{m}-\frac{1}{\Phi_{m}}\right) \text {. } \\
& {[d]_{m j}=\frac{1}{G_{s} J_{j} R_{j}}\left(\alpha_{j}-\frac{1}{\Phi_{j}}\right)} \\
& d_{m, m-1}=\frac{1}{G_{s} J_{m-1} R_{m-1} \Phi_{m-1}} \\
& d_{m, m+1}=\frac{1}{G_{s} J_{m} R_{m} \Phi_{m}}
\end{aligned}
$$

\section{（2）弾性方程式の荷重項}

a）等分布荷重

$$
\begin{aligned}
L_{m j}= & p_{j} \mathfrak{Q}_{1 j} \frac{R_{j}}{E_{s} I_{y^{\prime}, j}}\left[\frac{\left(1-\cos \Phi_{j}\right)\left(\Phi_{j}-\sin \Phi_{j}\right)}{2 \sin ^{2} \Phi_{j}}\right] \\
& +p_{j} \frac{R_{j}}{G_{s} J_{j}}\left[\mathfrak{Q}_{1 j}\left(\frac{\Phi_{j}}{2} \operatorname{cosec} \Phi_{j}-\frac{1}{2}\right) \tan \frac{\Phi_{j}}{2}\right. \\
& \left.-\mathfrak{\Omega}_{2 j}\left(\tan \frac{\Phi_{j}}{2}-\frac{\Phi_{j}}{2}\right)\right] \ldots \ldots \ldots \ldots \ldots \ldots(31) \\
N_{m j}= & \frac{p_{j}}{G_{s} J_{j}}\left[\mathfrak{Q}_{1 j}\left(\tan \frac{\Phi_{j}}{2}-\frac{1}{\alpha_{j}}\right)\right. \\
& \left.+\Omega_{2 j}\left(\frac{1}{\alpha_{j}}-\frac{\Phi_{j}}{2}\right)\right] \ldots \ldots \ldots \ldots \ldots \ldots \ldots(32)
\end{aligned}
$$

b）横方向線荷重

$$
\begin{aligned}
L_{m m}= & W_{m} \\
& \times\left[\frac{\mathfrak{\Omega}_{2 m}}{E_{s} I_{y^{\prime}, m}}\left(\frac{\psi_{m}{ }^{\prime} \sin \psi_{m}-\psi_{m} \sin \psi_{m}{ }^{\prime} \cos \Phi_{m}}{2 \sin ^{2} \Phi_{m}}\right)\right. \\
& +\frac{1}{G_{s} J_{m}}\left\{\mathfrak { Q } _ { 2 m } \left(\frac{\psi_{m}{ }^{\prime} \sin \psi_{m}-\psi_{m} \sin \psi_{m}{ }^{\prime} \cos \Phi_{m}}{2 \sin ^{2} \Phi_{m}}\right.\right. \\
& \left.\left.\left.-\frac{\sin \psi_{m}{ }^{\prime}}{\alpha_{m}{ }^{2} \sin \Phi_{m}}\right)-\mathfrak{\Omega}_{3 m}\left(\frac{\sin \psi_{m}{ }^{\prime}}{\sin \Phi_{m}}-\frac{\psi_{m}{ }^{\prime}}{\Phi_{m}}\right)\right\}\right]
\end{aligned}
$$




$$
\begin{aligned}
& L_{m, m-1}=W_{m-1}\left[\frac{\mathfrak{Q}_{2 m-1}}{E_{s} I_{y^{\prime}, m-1}}\right. \\
& \times\left(\frac{\psi_{m-1} \sin \psi^{\prime}{ }_{m-1}-\psi_{m-1}^{\prime} \sin \psi_{m-1} \cos \Phi_{m-1}}{2 \sin ^{2} \Phi_{m-1}}\right) \\
& +\frac{1}{G_{s} J_{m-1}}\left\{\mathfrak{\Omega}_{2 m-1}\right. \\
& \times\left(\frac{\psi_{m-1} \sin \psi_{m-1}^{\prime}-\psi^{\prime}{ }_{m-1} \sin \psi_{m-1} \cos \Phi_{m-1}}{2 \sin ^{2} \Phi_{m-1}}\right. \\
& \left.\left.\left.-\frac{\sin \psi_{m-1}}{\alpha^{2}{ }_{m-1} \sin \Phi_{m-1}}\right)-\mathfrak{L}_{s m-1}\left(\frac{\sin \psi_{m-1}}{\sin \Phi_{m-1}}-\frac{\psi_{m-1}}{\Phi_{m-1}}\right)\right\}\right] \\
& N_{m m}= \begin{cases}\frac{W_{m}}{R_{m} G_{s} J_{m}}\left(\mathfrak{Q}_{2 m} \frac{\sin \psi_{m}{ }^{\prime}}{\sin \Phi_{m}}-\mathfrak{Q}_{3 m} \frac{\psi_{m}{ }^{\prime}}{\Phi_{m}}\right) & \left(\psi_{m} \neq 0\right) \\
0 & \left(\psi_{m}=0\right)\end{cases} \\
& N_{m, m-1}=\left\{\begin{array}{cc}
\frac{W_{m-1}}{R_{m-1} G_{s} J_{m-1}}\left(\mathfrak{Q}_{2, m-1} \frac{\sin \psi_{m-1}}{\sin \Phi_{m-1}}\right. \\
\left.-\mathfrak{\Omega}_{3, m-1} \frac{\psi_{m-1}}{\Phi_{m-1}}\right) & \left(\psi_{m-1} \neq \Phi_{m-1}\right) \\
0 & \left(\psi_{m-1}=\Phi_{m-1}\right)
\end{array}\right.
\end{aligned}
$$

\section{(3) 断面力と変形量}

断面力と変形量は, 表一3 のとおりである。

\section{参考文 献}

1) 小西一郎 - 小松定夫 : 単純曲線桁橋 の立体的解析, 上木 学会論文集第 90 号, 昭和 38 年 2 月

2）小西一郎・小松定夫：薄肉連続曲線桁橋の立体的解析, 土木学会論文集第 91 号, 昭和 38 年 3 月

3）小松定夫：曲線並列桁橋の実用計算式, 土木学会論文集 第 93 号, 昭和 38 年 5 月

4) Sadao Komatsu : Structural Analysis for Continuous S-Shaped Curved Girder Bridge, Proc. 13th JNCAM, 1963

5) Ichiro Konishi and Sadao Komatsu : Three Dimensional Analysis of Curved Girder with Thin-Walled Cross Section, Pub. IABSE, Vol. 25, 1965
表一3 連続曲線箱桁の断面力と変形量

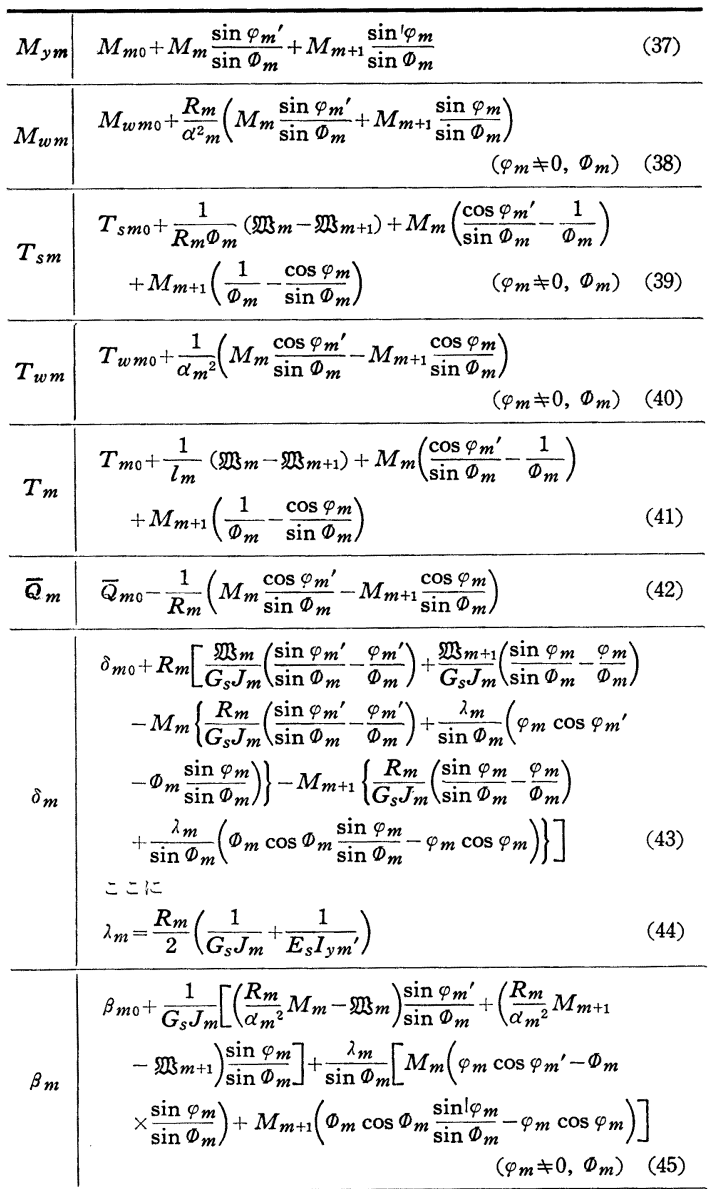

(1967.8.6 - 受付) 